\title{
Les ateliers du Matériel : pivots de la diffusion de l'organisation rationnelle au sein de la Société nationale des chemins de fer belges, 1910-1940
}

The workshops of the Equipment: fulcrum of the diffusion of the rational organization in the Belgian railways national society (SNCB), 1910-1940

\section{Éric Geerkens}

\section{OpenEdition Journals}

Édition électronique

URL : https://journals.openedition.org/rhcf/1765

DOI : 10.4000/rhcf.1765

\section{Éditeur}

Rails \& histoire

\section{Édition imprimée}

Date de publication : 1 décembre 2003

Pagination : 207-221

ISBN : 0996-9403

ISSN : 0996-9403

\section{Référence électronique}

Éric Geerkens, «Les ateliers du Matériel : pivots de la diffusion de l'organisation rationnelle au sein de la Société nationale des chemins de fer belges, 1910-1940», Revue d'histoire des chemins de fer [En ligne], 28-29 | 2003, mis en ligne le 19 décembre 2014, consulté le 22 avril 2022. URL : http:// journals.openedition.org/rhcf/1765; DOI : https://doi.org/10.4000/rhcf.1765

Ce document a été généré automatiquement le 22 avril 2022

Tous droits réservés 


\section{Les ateliers du Matériel : pivots de la diffusion de l'organisation rationnelle au sein de la Société nationale des chemins de fer belges, 1910-1940}

The workshops of the Equipment: fulcrum of the diffusion of the rational organization in the Belgian railways national society (SNCB), 1910-1940

Éric Geerkens

\section{Introduction}

1 En regard des pratiques observables dans l'industrie belge de l'entre-deux-guerres, la Société nationale des chemins de fer belges $(\mathrm{SNCB})^{1}$ apparaît comme une entreprise parmi les plus engagées dans l'adoption des méthodes d'organisation rationnelle du travail ${ }^{2}$. L'engouement pour ces méthodes est notamment illustré par la très forte présence de son cadre technique au sein du Comité national belge de l'organisation scientifique (CNBOS), auquel il fournit le plus gros contingent d'adhérents d'une même entreprise. Les ateliers du Matériel des Chemins de fer de l'État belge, puis de la SNCB, ont été à la fois le point d'entrée des méthodes d'organisation rationnelle du travail et, après l'étape concluante d'expérimentation, le point de diffusion de ces méthodes vers d'autres services de la société, mais aussi vers les constructeurs belges de matériel roulant. Pour comprendre ce processus, il convient d'abord de décrire les transformations de l'organisation du travail dans les ateliers de réparation et d'entretien du matériel roulant des Chemins de fer de l'État belge, puis de la SNCB, entre 1910 et 1940 environ. Il faut suivre ensuite le chemin de leur diffusion, au sein même du service du Matériel, puis vers d'autres activités ferroviaires. Enfin, l'intervention de la SNCB dans l'organisation des fabrications de ses fournisseurs de 
matériel roulant ne peut se comprendre dans les seuls termes de la diffusion du progrès technico-organisationnel; son étude renvoie à la constitution de la société, à la composition de son conseil d'administration et aux rapports entre secteurs public et privé.

\section{L'innovation organisationnelle au service du Matériel des Chemins de fer de l'État belge, puis de la SNCB}

2 Si les chemins de fer n'ont jamais manqué d'être organisés, l'adoption du système Rowan à l'Atelier central de Malines en 1910 peut être tenue pour le point de départ d'un large mouvement d'organisation qui repose sur l'étude détaillée du travail ouvrier dans les ateliers. Ce choix n'est pas propre à la Belgique : des études de temps avaient été conduites dans les ateliers des chemins de fer néerlandais et italiens à la veille de $1914^{3}$. Pour appliquer le système Rowan, qui encourage par une prime l'économie des temps d'exécution, il fallait établir une base de tarification des travaux à exécuter et déterminer des temps-étalons "pour des milliers de pièces"; une première forme d'analyse du travail ouvrier a donc été conduite à grande échelle. Celle-ci laisse cependant intacte la maîtrise des ouvriers sur l'organisation de leur travail, l'objectif consistant principalement à économiser du personnel en intensifiant l'effort ouvrier. Si les ouvriers et leurs représentants critiquent sévèrement le système Rowan, son application avant et après la guerre les « accoutume » à l'étude des temps, qui ne fera pas l'objet d'un rejet de principe.

3 L'étape suivante de réorganisation des ateliers se situe au lendemain de la guerre, dans un contexte marqué par la volonté d'industrialiser les Chemins de fer de l'État, qui aboutira à la constitution de la SNCB en $1926^{4}$. Même si on relève des références explicites à Taylor, cette phase de réorganisation s'inscrit d'abord et principalement dans le paradigme de la production en série - laquelle trouve dans le parc de wagons, voitures et locomotives un champ d'application particulièrement favorable - et a pour caractéristique principale une spécialisation des ateliers, en nombre progressivement réduit.

4 Ce sont les ateliers de moyenne réparation des locomotives qui, à partir de 1921, constituent le terrain d'expérimentation de la nouvelle organisation. En termes très généraux, formulés par le principal inspirateur de cette réforme, Narcisse Rulot ${ }^{5}$, la nouvelle organisation est caractérisée par "la spécialisation du travail et l'étude systématique de la production sous toutes ses faces ». Plus concrètement, cette organisation repose sur les principes suivants ${ }^{6}$ :

5 a. les travaux de réparation sont concentrés dans de grands ateliers affectés à la réparation d'un nombre limité de types de véhicules, ce qui permet d'abord la standardisation des pièces et la constitution de stocks d'éléments préfabriqués pour ces travaux ;

6 b. la spécialisation des ateliers s'est prolongée dans une répartition du personnel en brigades spécialisées en vue de travaux particuliers, et par une plus grande division horizontale du travail au sein de chaque brigade ;

c. les réparations sont effectuées selon une périodicité régulière, basée sur la longueur des parcours effectués; les travaux sont conduits suivant un programme-type étudié par la direction du Matériel et imposé à l'ensemble des ateliers ${ }^{7}$. 
8 Autour de 1925, la SNCB se dote également d'une véritable comptabilité industrielle, inspirée de celles du PLM et de la Compagnie du Nord; mais cinq ans plus tard, la comptabilité industrielle de certains ateliers ne paraît toutefois pas encore bien mise au point $^{8}$.

9 Entre 1922 et 1926, cette réorganisation a permis de réduire fortement la durée d'immobilisation des véhicules en réparation, qui passe de 67 à 26 jours; parallèlement le nombre moyen d'heures d'ouvriers se réduit de 3200 à 2100 heures par locomotive. La réparation des voitures à voyageurs et des wagons a été réorganisée, à partir de 1925 , suivant des principes comparables'.

10 Pour les différents types de matériel, la réduction des temps d'immobilisation permettait surtout de répondre à la demande de transport sans accroître excessivement le parc de matériel.

11 La constitution de la SNCB en 1926 va accélérer l'adoption des nouvelles méthodes d'organisation du travail, d'autant que les cadres de la société paraissent très réceptifs aux idées d'organisation scientifique, certes dans un contexte où ces idées bénéficient d'un large engouement. À partir de ce moment s'affirme une prépondérance du cadre technique sur celui des fonctionnaires de l'ordre administratif, l'organisation du travail relevant de "règles scientifiques que l'ingénieur dégage mieux et applique plus efficacement que le fonctionnaire administratif $»^{10}$.

12 Parmi les premières décisions prises par la SNCB figure la constitution d'un service, attaché à la direction du Matériel, qui est chargé d'étudier les questions d'organisation $\mathrm{du}$ travail et d'orientation professionnelle du personnel et qui publie rapidement des monographies décrivant les expériences d'organisation du travail à la SNCB.

13 Tout en poursuivant le mouvement de concentration et de spécialisation des ateliers amorcé sous le régime État, la SNCB s'engage, à partir de 1927, dans une véritable taylorisation de ses ateliers, en commençant cette fois par les ateliers de wagons, avant d'étendre la réforme à la réparation des voitures à voyageurs, puis des locomotives, suivant une progression qui va du matériel le plus simple au plus compliquée ${ }^{11}$.

14 Cette organisation repose sur l'étude puis la prescription du travail ouvrier: les directions des ateliers organisent la préparation du travail en faisant établir par des ouvriers, choisis parmi les plus compétents, des fiches de travail où sont consignés, pour chacun des travaux à effectuer, non seulement les temps d'exécution, mais surtout les procédés à employer et les outils à utiliser.

15 La séparation entre conception et exécution du travail va se matérialiser par la constitution, au sein des ateliers, de bureaux de fabrication chargés de la préparation du travail, de sa distribution et du contrôle de sa progression. L'activité de ces bureaux de fabrication contribue à standardiser les principes d'organisation du travail, tout en faisant bénéficier l'ensemble des ateliers de résultats d'études et de perfectionnements dans les méthodes de travail dégagés dans certaines sections d'atelier. Parallèlement, pour intensifier l'effort ouvrier, les ateliers substituent un régime de primes individuelles aux primes collectives; de même, le régime des primes du personnel de surveillance et de direction est revu pour encourager la réduction des prix de revient et de la durée d'immobilisation du matériel.

16 Le bilan de l'organisation nouvelle peut s'établir comme suit: entre la fin de l'année 1927 et la fin de 1930, l'effectif des ouvriers des ateliers centraux du matériel se réduit d'environ $14 \%$; en grande réparation, le nombre moyen d'heures de travail par 
locomotive réparée est en diminution de $31 \%$ et l'immobilisation moyenne passe de 69 à 50 jours quand, dans le même temps, le parcours moyen des locomotives entre deux réparations, indice de la qualité des travaux, s'allonge de $24 \%^{12}$. L'objectif de limitation de l'immobilisation du matériel de transport, poursuivi par l'amélioration de la qualité des travaux qui réduit la fréquence des passages du matériel en réparation, est à nouveau atteint.

17 À la taylorisation des ateliers s'ajoute, à partir de 1930, l'organisation du travail à la chaîne, adoptée pour le placement de freins à air sur les wagons du service international ${ }^{13}$. Selon le directeur du Matériel : «le travail à la chaîne [...] fut l'origine d'une transformation radicale de nos méthodes. Nous y trouvâmes la véritable clef de tous nos problèmes de fabrication et de réparation du matériel roulant ${ }^{14}$. » Suivant l'exemple du fliessarbeit allemand ${ }^{15}$, la direction du Matériel aménage, à l'atelier central de Louvain, des chaînes à avancement intermittent pour y équiper, en quatre ans, 60000 (en fait 48000 ) wagons, traités par lots de séries identiques, ce qui en fait le programme d'installation des freins à air le plus rapide de tous ceux suivis par les réseaux européens ${ }^{16}$. Alors que certaines compagnies de chemin de fer se voyaient réclamer près de 80 heures de travail pour l'équipement d'un wagon, l'organisation adoptée à Louvain permet de réduire ce temps à 22 heures. Outre cette économie directe, l'objectif principal de l'organisation à la chaîne est à nouveau la limitation de l'immobilisation du matériel en cours de transformation, auquel s'ajoute une perspective d'économie sur l'emploi des quelque 3000 serre-freins.

Forte de cette expérience - qui agit comme un véritable révélateur ${ }^{17}$ - la SNCB va étendre le travail à la chaîne à d'autres travaux exécutés par le service du Matériel. Ainsi, cette technique, adoptée pour une, puis plusieurs opérations ponctuelles (freins Westinghouse, éclairage électrique, pose de cendriers étanches, etc.), va être appliquée aux travaux récurrents de réparation et d'entretien du matériel roulant, champ d'application moins aisé en raison de la variété du matériel et des avaries qui l'affectent. En 1931, la SNCB estime que l'application du travail à la chaîne permet une économie totale de l'ordre de $20 \%$ par rapport à l'organisation ancienne. L'économie procurée par cette organisation autofinance les investissements que nécessite la transformation des ateliers pour le travail à la chaîne.

Dès le début de la crise, la baisse du trafic de marchandises et la dégradation de ses résultats financiers ont conduit la SNCB à approfondir la rationalisation des différents travaux, par une combinaison de techniques déjà éprouvées et de procédures nouvelles, visant plus particulièrement la planification et le contrôle.

Parmi les premières, la taylorisation - processus long et progressif - va être étendue, essentiellement par un élargissement du champ d'intervention des bureaux de fabrication, par une meilleure préparation du travail reposant sur l'établissement de feuilles de visite, sorte de nomenclature des avaries, et par la constitution d'un service de chronométrage, permettant de resserrer les temps opératoires. Les feuilles de visite, comme instruments de saisie de l'information, vont faire le lien entre la préparation du travail et le contrôle de son exécution ${ }^{18}$. L'extension du travail à la chaîne s'accompagne d'une standardisation accrue des matériaux, formes et dimensions et d'un développement des procédures de contrôle de l'exécution du travail, organisées par un service créé à cet effet.

21 Parmi les techniques nouvelles, le planning et le contrôle budgétaire permettront une surveillance plus étroite de l'activité et des dépenses des ateliers. Si des formes de 
contrôle de l'exécution des programmes de travail existaient déjà à la fin des années 1920, de véritables bureaux de planning sont insérés, en 1932, dans l'organigramme des ateliers de réparation de locomotives, entre les bureaux de fabrication et les services d'exécution. Différents types de planning en gigogne assurent à la fois le respect des délais inscrits dans les programmes de réparation et l'occupation la plus complète du personnel des ateliers. La SNCB a été très tôt informée de l'intérêt du contrôle budgétaire, puisque son directeur des finances représentait la Belgique à la Conférence internationale sur le contrôle budgétaire, tenue à Genève en juillet 1930. La direction du Matériel recourt à cette technique pour exercer une surveillance centralisée des activités de réparation des locomotives, sur une base non plus technique mais financière ${ }^{19}$.

L'emploi de ces techniques d'organisation dans la première moitié des années 1930 se traduit par les résultats suivants :

23 - entre 1931 et 1935, le nombre moyen d'heures de travail consacrées à la grande réparation de locomotives accuse une nouvelle diminution de $22 \%{ }^{20}$ et son coût global à prix constants baisse de $12,3 \%$ entre 1931 et $1934^{21}$;

24 - pour la réparation des wagons, la productivité du travail s'accroît dans des proportions qui font écrire que « un homme de 1933 donne une production équivalente à celle de trois hommes de $1929 »^{22}$;

25 - l'effectif total du service du Matériel se réduit de $22 \%$ entre 1931 et 1935, celui des ateliers centraux de $38 \%$, alors que dans le même temps l'ensemble du personnel de la $\mathrm{SNCB}$ ne se contracte que de $13 \%$. En période de crise, à la limitation de l'immobilisation s'ajoute une volonté, plus marquée qu'auparavant, de comprimer les effectifs.

\section{La diffusion de l'organisation expérimentée par la direction du Matériel}

Les différents principes d'organisation éprouvés à la direction du Matériel vont être étendus aux activités d'autres directions de la SNCB, puis, pour certains d'entre eux, être imposés aux fournisseurs de matériel roulant.

\section{Au sein de la SNCB}

L'organisation mise au point au milieu et à la fin des années 1920 a été introduite dans les ateliers de réparation du service de la Voie, dont les activités ne sont pas de nature bien différente de celles des ateliers du Matériel: concentration, recherche des possibilités de travail en série, constitution d'un bureau de fabrication, intensification du travail par un régime de primes ${ }^{23}$.

Une taylorisation des activités administratives de l'ensemble de la SNCB est entreprise à partir de $1938^{24}$.

29 La SNCB va appliquer les principes du travail à la chaîne à d'autres activités que la réparation et l'entretien du matériel. Au service de l'Exploitation, la recherche d'un équilibre optimal entre les postes successifs, déterminant majeur du rendement dans le travail à la chaîne, fournit le principe d'une organisation nouvelle du triage des wagons ${ }^{25}$. De même, les travaux d'entretien des voies ont été réorganisés de manière à 
les faire exécuter suivant certains principes du travail à la chaîne où, sans convoyeur, les travailleurs se déplacent par rapport à un chantier fixe et exécutent des opérations nettement circonscrites et coordonnées dans le temps. C'est en pensant à une organisation du travail comparable - «où les éléments de travail sont constitués par des chantiers fixes : c'est alors l'ouvrier qui est mobile et répète, sur chacun d'eux, des opérations strictement limitées et standardisées »- que G. Friedmann a proposé une définition élargie du travail à la chaîne ${ }^{26}$.

Comme elle l'avait fait pour le travail à la chaîne, la société va étendre l'usage du planning à l'ensemble des ateliers du Matériel, puis la faire appliquer ensuite à d'autres activités ferroviaires, comme l'entretien des voies ou la fabrication de matériel pour la voie $^{27}$.

\section{Vers les constructeurs belges de matériel roulant}

La SNCB innove également dans un domaine très curieux dans ce sens qu'elle impose la rationalisation à ses fournisseurs. Ayant à remettre une commande de 1000 wagons à marchandises et constatant que les prix qui lui étaient soumis étaient trop élevés, elle a fait étudier par ses ingénieurs la rationalisation de la construction des wagons et, se faisant l'instructeur de ses fournisseurs, a pu leur démontrer que le nombre total d'heures d'ouvriers nécessaires pour la construction d'un wagon pouvait être ramené de 4300 à 2900 .

Bull. I.O.S.T., septembre 1931, vol. V, n 9, p. 199.

La SNCB a également imposé à certains de ses fournisseurs l'emploi de techniques d'organisation qu'elle avait expérimentées, son intérêt lui paraissant rencontrer celui des fabricants de matériel roulant. La direction de la SNCB considère en effet que cette industrie ne peut survivre qu'en obtenant d'importantes commandes à l'exportation, à la mesure notamment de la part réservée à la Belgique dans le Cartel international des wagons ( $34,6 \%$ contre $28,8 \%$ à l'Allemagne et $13,9 \%$ à la France). Cet objectif implique un abaissement des prix de revient des fabrications et donc «l'application impitoyable des méthodes de rationalisation ". Se prévalant d'une expérience probante en matière d'organisation, la SNCB pense avoir " pour devoir et pour intérêt d'aider l'industrie à se moderniser $\aleph^{28}$. La pratique des chemins de fer allemands la conforte dans ses exigences à l'égard des constructeurs ${ }^{29}$.

Pour comprendre le rôle tenu en cette matière par la $\mathrm{SNCB}$, il convient de rappeler ici que celle-ci a été conçue en 1926 dans le giron de la principale holding belge : la Société Générale de Belgique et qu'elle est dirigée par un conseil d'administration où siègent des représentants des principaux groupes industriels belges. Le banc patronal est « conduit » par Alexandre Galopin, directeur de la division de l'Industrie de la Société Générale de Belgique, qui est également membre du comité permanent de la SNCB et représente cette dernière comme employeur à la commission paritaire créée en son $\operatorname{sein}^{30}$. L'influence de la Générale se marque aussi par le rappel, à la direction du Matériel, de l'inspirateur de la concentration et de la spécialisation des ateliers dans l'après-guerre, Narcisse Rulot, parti diriger l'exploitation de la Compagnie du chemin de fer du Congo ; rappelons que Rulot deviendra le directeur général de la SNCB en 1932.

La SNCB a imposé le travail à la chaîne à ses fournisseurs de matériel roulant. A cette fin, elle a abandonné le principe ancien d'adjudication en petits lots dispersés - qui la laissait désarmée face à l'entente des constructeurs - pour n'offrir que des séries plus 
importantes, et n'admettre comme soumissionnaires qu'un nombre limité de candidats, équipés pour le travail en série.

Ainsi, devant faire l'acquisition d'un millier de wagons en 1931, la SNCB a réparti cette fourniture en quatre lots et a imposé aux adjudicataires la remise d'un devis comprenant le plan des chantiers de montage et une estimation de la main-d'œuvre nécessaire. Comme le précise la citation en exergue, la SNCB va examiner ces documents à l'aune de son expérience du travail à la châne et faire réduire fortement (33\%) le nombre d'heures nécessaires à la fabrication d'un wagon; par rapport au marché précédent, datant de 1929, le prix du wagon (à prix courants) baisse de $\pm 22 \%{ }^{31}$.

En 1931 toujours, dans le cadre d'une importante adjudication de voitures métalliques à voyageurs, la SNCB va intervenir encore plus directement dans l'organisation du travail des constructeurs en leur dictant leur méthode de travail - travail à la chaîne avec avancement obligatoire et sous-chaînes synchrones - et en exigeant, outre les plans des chaînes, les fiches de travail et les temps d'exécution de chaque opération. La confrontation de ces documents avec les devis établis pour ces mêmes travaux dans les ateliers de la SNCB permet à nouveau de faire baisser le nombre d'heures initialement prévues (de $20 \%$, puis de $31 \%$ au stade de l'exécution de la commande). En marge de ce dispositif de compression des prix, les ingénieurs de la SNCB ont aidé leurs homologues du secteur privé à mettre au point leurs chaînes de montage, en particulier à décomposer le travail en postes équilibrés d'égale durée. Dans le cadre de cette commande, la SNCB a par ailleurs mis en adjudication certaines pièces et organes de voiture, en excluant les constructeurs agréés pour les voitures, de manière à favoriser à nouveau les productions en grandes séries spécialisées.

La société a également imposé l'emploi des plannings aux constructeurs de matériel roulant, ce qui lui permet notamment de surveiller les délais de fourniture de son matériel.

La SNCB intervient ainsi dans un domaine, l'organisation du travail, à propos duquel les employeurs ne cessent de rappeler - s'adressant certes aux représentants syndicaux qu'il s'agit d'une prérogative patronale; or, les ingénieurs de la SNCB vont véritablement s'immiscer dans l'organisation des fabrications de ses fournisseurs, pour la contrôler et la corriger.

On observe donc ici des rapports originaux entre une entreprise publique et le secteur privé. Le conseil d'administration des chemins de fer engage la société à expérimenter des mesures d'organisation novatrice; pour ce qui concerne le travail à la chaîne, les ateliers du Matériel de la SNCB devancent nettement les constructeurs belges de matériel roulant. Ensuite les commandes de cette entreprise publique - soutenue en période de crise par une importante intervention financière de l'État - vont en quelque sorte financer la réorganisation interne de certaines activités des constructeurs privés, suivant l'expérience de la SNCB. La volonté rationalisatrice de l'entreprise publique, dont l'intervention chez les constructeurs lui paraît probante, ne rencontre toutefois pas entièrement la logique productive de ces derniers. Ainsi, une de ces entreprises de constructions métalliques (Ateliers métallurgiques de Nivelles), qui n'était pas entièrement spécialisée dans la fabrication de matériel de chemin de fer (aucune ne l'était complètement), se plaint précisément que l'organisation imposée par la SNCB " désorganise complètement un atelier " $^{32}$.

Pour comprendre cette expérience, surprenante à l'aune de nos représentations autant qu'à celles des contemporains, pour la comprendre sans se méprendre sur sa portée, il 
convient de rappeler l'importante présence patronale au conseil d'administration de la $\mathrm{SNCB}$, dont la délégation est dirigée par Galopin. Or ce dernier préside dans le même temps le conseil d'administration des deux principaux constructeurs belges de matériel roulant (Ateliers métallurgiques de Nivelles et La Brugeoise et Nicaise et Delcuve), qui sont les adjudicataires les mieux placés, et qui se renforcent encore parce qu'ils sont adjudicataires ${ }^{33}$. On peut donc penser que la représentation du secteur privé dans le secteur public a favorisé le soutien public à la réorganisation du privé, avec un subside étatique qui relève d'abord d'une politique de grands travaux, destinée à remettre au travail un grand nombre de chômeurs.

\section{En guise d'épilogue : une première réflexion de la direction de la SNCB sur les limites de la rationalisation}

Reconnaissant des emprunts à divers réseaux étrangers (principalement français et allemand), la SNCB a inséré dans son programme de concentration et de spécialisation des ateliers du matériel diverses pratiques d'organisation, alors tenues pour les plus rationnelles (taylorisation, travail à la chaîne, planning...). Partant des ateliers du Matériel, l'emploi de ces techniques d'organisation a été étendu de manière systématique dans l'ensemble de la société. En présentant ces applications dans la presse technique et en les imposant à ses fournisseurs, la SNCB a été un puissant vecteur de diffusion de ces méthodes d'organisation dans l'industrie belge.

41 Ses dirigeants semblent parmi les premiers à formuler une réflexion critique sur les limites de leur pratique rationalisatrice, limites qui tiennent principalement à la forte concentration des pouvoirs de décision au sommet de la hiérarchie administrative, prolongeant une division verticale du travail très marquée. Au terme de la période étudiée ici, la direction de la société a expliqué que cette extrême centralisation de la décision avait été une condition indispensable de réussite de la rationalisation:

Les méthodes d'exploitation en vigueur sous le régime «État belge » n'avaient pas éduqué l'ensemble du personnel dans cet esprit ; la Société était contrainte dès lors, de centraliser dans les mains de quelques fonctionnaires spécialement qualifiés et relativement peu nombreux, les tâches nouvelles à exécuter. La centralisation était donc, en raison des circonstances spéciales dans lesquelles la SNCB avait repris l'héritage de l'État, une condition indispensable de réussite ${ }^{34}$.

Cette centralisation s'est inscrite dans les structures de la SNCB. Une bonne illustration en est donnée par l'entretien des quelque 105000 wagons : tous les ateliers sont pilotés par le Service central des wagons de la direction du Matériel à Bruxelles. Si la centralisation était une condition d'application réussie des nouvelles méthodes d'organisation, elle était porteuse de conséquences quelque peu dommageables. Ce qui est en cause c'est d'abord la mauvaise coordination entre les services d'exécution au niveau local, dont les rapports sont régis par la voie hiérarchique ; mais c'est surtout " la mauvaise répartition des responsabilités entre tous les fonctionnaires dirigeants ", en clair une sous-utilisation, au niveau local, des ingénieurs dont le travail s'appauvrit. Cette critique anticipe de plusieurs décennies, certes pour une catégorie nettement supérieure de personnel, la mise en cause de la perte d'autonomie et d'initiative ouvrière qu'induit le taylorisme. Ce bémol particulier mis au credo rationalisateur est 
peu ordinaire dans le monde des ingénieurs dirigeants d'entreprise à la fin de l'entredeux-guerres.

\section{NOTES}

1. Pour situer l'importance du réseau $\mathrm{SNCB}$, rappelons quelques ordres de grandeur, relatifs à l'année 1938 : la longueur des voies atteint $4838 \mathrm{~km}$, le matériel se compose de 3181 locomotives à vapeur et de 67 automotrices, de 8017 voitures et 106848 wagons. Les effectifs totaux en activité comptent alors 71472 travailleurs, dont 20160 sont occupés à la Voie, 27261 à la Traction (et Matériel), 23471 à l'Exploitation, et enfin 580 dans les Services généraux. Le volume du trafic s'établit, en 1938, à 5965 millions de voyageurs $/ \mathrm{km}$ et à 5143 millions de tonnes $/ \mathrm{km}$.

2. É. Geerkens, La Rationalisation dans l'industrie belge de l'Entre-deux-guerres, Liège, université de Liège, thèse de doctorat en histoire, 2002. Nous avons déjà présenté les choix d'organisation posés par la SNCB avant la Seconde Guerre mondiale, et les réactions suscitées dans les rangs cheminots : É. Geerkens, « La rationalisation dans l'industrie belge : les réalisations de la Société nationale des chemins de fer belges (1926-1940) ", Revue belge de philologie et d'histoire, 76-2, 1998, p. 443-500; nous avons élargi le champ de cette étude dans: É. Geerkens, "Structures et organisation de la SNCB dans une perspective historique", in B. van der Herten; M. van Meerten; G. Verbeurgt (sous la dir. de), Le Temps du train. 175 ans de chemins de fer belges. $75^{e}$ anniversaire de la SNCB, Louvain, Presses universitaires de Louvain, 2001, p. 241-271. En termes de documentation relative à la question de l'organisation du travail dans les ateliers des chemins de fer belges, deux situations doivent être distinguées. Les archives des Chemins de fer de l'État belge sont particulièrement pauvres, ce qui impose le dépouillement de sources indirectes (publications officielles, notes de cours, presse syndicale, etc.). Outre le recours à la presse technique et syndicale, le régime SNCB peut être étudié à partir des archives du secrétariat du conseil d'administration, en particulier de la collection des documents techniques soumis au conseil; par contre, le service du matériel ne semble pas avoir conservé d'archives très anciennes. Précisons que l'organisation du travail au sein des compagnies exploitant des lignes concédées (dont le Nord belge), ou vicinales, n'est pas abordée dans cette contribution.

3. Bloemen, E.S.A., Scientific Management in Nederland. 1900-1930, Amsterdam, NEHA série III, 1988, p. 76, note 37. En Italie, les études préliminaires à l'introduction du système Rowan aux Ferrovie dello Stato ont été conduites à partir de 1914, mais le système n'a été introduit qu'au lendemain de l'Armistice, G. sapelli, Organizzazione, lavoro e innovazione industriale nell'Italia tra le due guerre, Turin, Rosenberg et Sellier, 1978, p. 82-83.

4. Sur les projets d'industrialisation des Chemins de fer de l'État belge et la constitution de la $\mathrm{SNCB}$, voy. G. Vanthemsche, «L'Entre-deux-guerres, période charnière de l'histoire des chemins de fer belges (1919-1939) », in B. Van der Herten; M. Van Meerten; G. Verbeurgt, op. cit., p. 151-176.

5. Narcisse Rulot (1883-1978), ingénieur civil diplômé de l'École des Mines de Liège (1905), est entré au service des Chemins de fer de l'État belge en 1906, il est attaché à la direction du Matériel en 1920 ; cette carrière n'a été interrompue qu'en [1924]1926 lorsqu'il fut nommé à la direction de l'Exploitation de la Compagnie du chemin de fer du Congo. En 1928, il est rappelé en Belgique pour diriger le service du Matériel de la SNCB, puis la Société elle-même, entre décembre 1932 et 1945 ; il est alors présenté comme le premier directeur du Matériel à accéder à 
la direction générale dans l'histoire des chemins de fers publics belges, Feuille d'avis de l'A.I.Lg, $\mathrm{n}^{\circ} 2$, février 1956, p. $37 ; \mathrm{n}^{\circ} 12$, décembre 1960, p. 333. S'agissant d'activités d'exploitation de chemins de fer, le rôle personnel d'un ingénieur ne doit pas être surestimé ; comme l'écrit Rulot lui-même, «le caractère spécial des exploitations de chemins de fer est de n'avoir pas de secret, de publier leurs inventions, de les répandre même chez les autres chemins de fer ", N. Rulot, L'Organisation du travail à la chaîne dans les ateliers de la Société nationale des chemins de fer Belges, Bruxelles, CNBOS (public. n 3), 1931, p. 23-24. L'état des sources disponibles nous expose plutôt au risque de ne retenir que quelques grands noms, dont ceux de Rulot et Jadot, et d'ignorer le rôle tenu par d'autres ingénieurs (comme Burton, Verkoyen, Warte, etc.) dans la transformation des méthodes d'organisation.

6. Ces principes ont été détaillés dans les enseignements de N. Rulot (avec la collaboration de E. Hennig et A. Chantrell), Exploitation du service de traction des trains. Cours de l'École nationale des chemins de fer à l'usage des ingénieurs, des fonctionnaires et des agents de surveillance des remises, Bruxelles, s.d. (1923-1928) [fac-similé reproduit par J. Vandenberghen dans la série Musée des chemins de fer belges, SNCB-département Matériel. XIII. Période 1919-1926, nº 4].

7. Cet entretien systématique est comparable, mutatis mutandis, à l'entretien des voies par révisions générales ou méthodiques, que la SNCB adopte alors suivant le modèle de la Compagnie des chemins de fer du Nord, cf. R. Campus, «L'entretien méthodique des voies de chemin de fer », Annales de l'Association des ingénieurs sortis des écoles spéciales de Gand, 1928, nº 4, p. 481-535.

8. É. Geerkens, «La rationalisation... ", art. cité, p. 448, note 18 ; F. Delory, « Le premier quart de siècle de la SNCB ",Trains, octobre 1951, n spécial, 3-4, p. 4 ; E. Desorgher, «Les finances ", ibidem, p. 89 ; P. Dufour et A.W. Chantrell, « La nouvelle organisation des Ateliers centraux de Salzinnes (Namur) », Bulletin de l'Association Internationale du Congrès des Chemins de fer (désormais Bull. AICF), octobre 1929, n 10, p. 1917-1918.

9. Rapport sur le service de la traction et du matériel pendant l'année 1924, Bruxelles, s.d., p. 20-21, 27, 37-38; N. Rulot (en collaboration avec E. Hennig et A. Chantrell), op. cit., p. 799.

10. SNCB, Documents soumis au conseil d'administration (désormais Doc. C.A.), Réorganisation du contrôle des recettes, doc. $\mathrm{n}^{\circ}$ 23, 15 octobre 1927, p. 3-4 ; SNCB, Doc. C.A., Nomination d'ingénieurs au service de l'exploitation, doc. $n^{\circ} 24,15$ octobre 1927, p. 1-3.

11. $M$. Warte, «Réorganisation de l'entretien et des réparations du matériel de transport à marchandises ", Bull. AICF, janvier 1930, XII, n 1, p. 2-18; SNCB, Doc. C.A., Réorganisation des ateliers de wagons et de voitures, doc. $\mathrm{n}^{\circ} 301,14$ janvier 1929 ; SNCB, Procès-verbal de la réunion du Conseil d'Administration de la SNCB du 26 avril 1929, C.A. $\mathrm{n}^{\circ}$ 35, p. 5 ; SNCB, Doc. C.A., Organisation du contrôle du travail dans les ateliers de réparation de locomotives, doc. $\mathrm{n}^{\circ} 360,23$ avril 1929, p. 2 ; P. Dufour et A.W. Chantrell, op. cit., p. 1916-1925.

12. SNCB, Rapports sur l'exploitation..., exercices 1926-1927 à 1930, en particulier exercice 1928, p. 80 et exercice 1930, p. 92 ; SNCB, Doc. C.A., Concentration des moyennes réparations des locomotives, doc. $\mathrm{n}^{\circ} 857,31$ juillet 1931, p. 9 [ce document traite des moyennes et grandes réparations].

13. Dans une perspective historique, ces techniques d'organisation se superposent bien plus qu'elles ne se suivent et participent dans leur interdépendance à l'organisation d'ensemble des ateliers. Ainsi, le travail en série a justifié à la fois l'étude et la prescription du travail autant que le travail à la chaîne ; celui-ci réclame et permet une « meilleure taylorisation » du travail ouvrier et l'emploi économiquement justifié d'un outillage spécialisé à grand débit, il impose également des études de tolérance et la standardisation des matériaux, formes et dimensions...

14. N. Rulot, L'Organisation du travail à la chaîne..., op. cit., p. 2.

15. À propos du fliessarbeit d'inspiration fordienne des chemins de fer allemands, voy. J.-H. Peters, «Rationalisierungsbestrebungen der Deutschen Reichsbahn-Gesellschaft zwischen 1924 und 1929 », Zeitschrift für Unternehmensgeschichte, 1996, n² 2, p. 187-200, spéc. p. 196. 
16. SNCB, Doc. C.A., Application du frein Westinghouse aux trains de marchandises. Mesures d'exécution, doc. $\mathrm{n}^{\circ} 375,16$ mai 1929; SNCB, Doc. C.A., Choix des constructeurs à agréer pour la prochaine adjudication des wagons, doc. $\mathrm{n}^{\circ} 729,18$ février 1931, p. 8.

17. Ainsi, en comparant le coût d'une transformation qu'elle fait exécuter à la chaîne dans ses propres ateliers avec le coût de cette même transformation, certes opérée sur une série beaucoup plus courte, réalisée dans l'industrie privée un an plus tôt, elle constate une baisse de prix d'environ $45 \%$, SNCB, Doc. C.A., Installation de l'éclairage électrique sur 1000 voitures par l'Atelier central de Malines, doc. $\mathrm{n}^{\circ}$ 675, 13 décembre 1930.

18. Sur l'importance de cet instrument d'organisation, voy. F. Arnould, Planning, rôle du planning dans les ateliers de réparation du matériel roulant de la SNCFB, Liège, mémoire de fin d'études de l'Institut Gramme, 1948, p. 25-26.

19. G. Jadot, Le Contrôle et la gestion des entreprises à l'aide du Budget, Bruxelles, CNBOS (public. $\mathrm{n}^{\circ} 2$ ), 1931 ; H.J.H. Challe, « Application du contrôle budgétaire aux travaux de réparation », Bull. CNBOS, février 1936, n² 2, p. 29-33; mars 1936, n 3, p. 65-68.

20. Entre 1927 et 1939, le nombre moyen d'heures d'ouvrier par locomotive réparée (grande réparation) passe de 9204 à 4 318, soit une diminution de $53 \%$.

21. A. Verbeemen, «Réorganisation des services des Ateliers centraux de Malines (Société nationale des chemins de fer belges) », Bull. AICF, mai 1934, n 5, p. 468-469.

22. G. Op't Eynde, «L'organisation de la réparation des wagons à l'Atelier central de Gentbrugge de la SNCB », Bull. CNBOS, octobre 1935, n 10, p. 229-230.

23. E. Desorgher, «Quelques aspects de l'activité d'un dépôt central du service de la voie de la SNCB ", Annales de l'Association des ingénieurs sortis des écoles spéciales de Gand, 1933, $\mathrm{n}^{\circ} 4$, p. 411-432 ; C. Lemaire et J. Schotte, " Note sur la fabrication de pièces moulées en béton armé », Bull. AICF, octobre 1931, n 10, p. 843-867 ; SNCB, Doc. C.A., Annexe au procès-verbal n ${ }^{\circ} 143$ de la séance du Comité permanent du 11 avril 1930, doc. n561, s.d., p. 5.

24. E. Desorgher, «Association internationale du Congrès des chemins de fer. Étude de questions d'intérêt général. Question II : Quel est l'aspect sous lequel, du point de vue de l'intérêt général comme de celui des réseaux de chemins de fer, doivent être dorénavant envisagés les problèmes de simplification dans l'exploitation. Rapport (Belgique et pays de langue anglaise) », Bull. AICF, mars 1939, $n^{\circ}$ 3, p. 204 ; Centre d'organisation de la Société nationale des chemins de fer belges, "L'organisation de la sténo-dactylo à la SNCFB », in Le Rendement du travail de bureau, Bruxelles, CNBOS (public. $n^{\circ} 38$ ), 1941, p. 93-98; «L'organisation et la mesure du rendement au Central mécanographique de la SNCFB », ibidem, p. 103-110.

25. F. Dessent et J. Colle, « Application du travail à la chaîne à la gare de formation d'Anvers-Nord de la Société nationale des chemins de fer belges ", Bull. AICF, novembre 1931, n 11, p. 941-943; SNCB, Doc. C.A., Application du travail à la chaîne à la gare d'Anvers-Nord, doc. nº 834, 9 juillet 1931.

26. G. Friedmann, Où va le travail humain ?, Paris, Gallimard, 1950, p. 228.

27. R. Campus, La Réalisation d'une voie ferrée de qualité. Les résultats techniques, économiques et sociaux de quinze années d'organisation, Bruxelles, M. Hayez, 1946, p.121-126; L. Lhoest, "Application du planning au service de la voie», Bull. technique de l'Union professionnelle des inspecteurs techniques et chefs de section des chemins de fer belges, 15 septembre 1935, $\mathrm{n}^{\circ}$ 58, p. 19-22 ; Musée des chemins de fer belges-SNCB, Doc. 86.105. 8, SNCB, Service de la Voie. Circulaire $\mathrm{n}^{\circ} 6 \mathrm{du}$ Bureau 32-3 à MM. les ingénieurs principaux des sept groupes, Planning des travaux d'entretien des voies, Bruxelles, le 20 février 1936.

28. SNCB, Doc. C.A., Choix des constructeurs..., doc. $\mathrm{n}^{\circ} 729$, op. cit., p. 1-2.

29. SNCB, Doc. C.A., La Voiture métallique des chemins de fer allemands, doc. n 818, 13 juin 1931.

30. G. Kurgan-van Hentenryk, « Alexandre Galopin », in G. Kurgan-van Hentenryk ; S. Jaumain ; V. Montens (sous la dir. de), Dictionnaire des patrons en Belgique. Les hommes, les entreprises, les réseaux, Bruxelles, De Boeck Université, 1996, p.301-303. Sur le rôle particulier de Galopin à la 
commission paritaire de la SNCB, voy. D. Luyten, «La SNCB en tant qu'employeur », in B. Van der Herten ; M. van Meerten ; G. Verbeurgt (sous la dir. de), op. cit., p. 275-280.

31. SNCB, Doc. C.A., Nature des marchés relatifs à la construction des voitures métalliques, doc. $\mathrm{n}^{\circ} 2284$, $1^{\mathrm{er}}$ juillet 1935, p. 1.

32. Ministère de l'Emploi et du Travail, Secrétariat du service des relations collectives du travail, 111. Comité national de la construction mécanique, 5 janvier 1931-24 avril 1934, séance du 18 avril 1934, p. 11.

33. Le rôle de la Société Générale dans l'adjudication des voitures à voyageurs sera dénoncé à la Chambre : «[...] la politique pratiquée en fait par la Société nationale sert admirablement la politique de concentration poursuivie par la Société Générale et la sert au moyen des deniers de la collectivité et par des procédés purement artificiels ", Ann. parl., Ch. Repr., sess. ord. 1931-1932, séance du 22 décembre 1931, p. 419 (intervention du député démocrate-chrétien Bodart).

34. SNCB, Doc. C.A., Question $n^{\circ} 11$, Réforme de structure de l'organisation des services de la SNCB, 30 juin 1942, p. 2. Ce mouvement de centralisation s'est approfondi tout au long des années 1930, cf. SNCB, Doc. C.A., Attribution des groupes du matériel, doc. $n^{\circ} 2$ 461, 18 décembre 1935.

\section{RÉSUMÉS}

En regard des pratiques observables dans l'industrie belge de l'entre-deux-guerres, la Société nationale des chemins de fer belges ( $\mathrm{SNCB}$ ) apparaît comme une entreprise parmi les plus engagées dans l'adoption des méthodes d'organisation rationnelle du travail. Les ateliers du Matériel des Chemins de fer de l'État belge, puis de la SNCB, ont été à la fois le point d'entrée des méthodes d'organisation rationnelle du travail et, après l'étape concluante de l'expérimentation, le point de diffusion de ces méthodes vers d'autres services de la société, mais aussi vers les constructeurs belges de matériel roulant.

Pour comprendre ce processus, l'article s'attache d'abord à décrire les transformations de l'organisation du travail dans les ateliers de réparation et d'entretien du matériel roulant des Chemins de fer de l'État belge, puis de la SNCB, entre 1910 et 1940 environ. Ensuite, l'auteur retrace le chemin de leur diffusion, au sein même du service du Matériel, puis vers d'autres activités ferroviaires. Enfin, l'intervention de la SNCB dans l'organisation des fabrications de ses fournisseurs de matériel roulant ne peut se comprendre dans les seuls termes de la diffusion du progrès technico-organisationnel; son étude renvoie à la constitution de la société, à la composition de son conseil d'administration et aux rapports entre secteurs public et privé.

En conclusion, l'auteur insiste sur le problème de la centralisation extrême qui accompagna l'organisation rationnelle du travail, centralisation du travail des cadres perçue par certains acteurs dès les années 1940 comme à la fois nécessaire à la réussite éclatante du système mais porteuse d'inconvénients (mauvaise coordination horizontale, déresponsabilisation des agents) qui firent, plus tard, l'objet d'une contestation par le monde ouvrier.

Next to observable practices in the Belgian industry of the inter-war period, the Société nationale des chemins de fer belges ( $\mathrm{SNCB}$ ) appears as a company among the most engaged in the adoption of rational organization of work methods. The workshops of the Equipment of the Belgian State railways (and then the $\mathrm{SNCB}$ ) were both the entry point of these methods and, after the conclusive stage of the experiment, the diffusion point of them to other services of the company as well as to Belgian manufacturers of rolling stock. To understand this process, the article first 
describes changes in the organisation of labour in the rolling stock repair and maintenance workshops of the Belgian State (then SNCB) between 1910 and 1940.

Afterwards, the author traces the path of their dissemination within the Equipment service and then to other railway activities.

Finally, the intervention of the SNCB in the manufacturing organisation of its suppliers of rolling stock cannot be understood only in terms of dissemination of technical-organizational progress. its study refers to the incorporation of the company, to the composition of its Board of Directors and the relationship between public and private sectors.

In conclusion, the author insists on the problem of extreme centralization that accompanies the rational organization of labour. The executives work's centralization was indeed as soon as the 1940's perceived by some actors as both necessary to the outstanding success of the system and carrying disadvantages (poor horizontal coordination, disempowerment of agents). Facts that would later be challenged by the workers' world.

\section{INDEX}

Mots-clés : atelier, Belgique, entre-deux-guerres, histoire sociale, innovation, organisation du travail, rationalisation du travail

Keywords : Belgium, innovation, interwar period, organization of labor, social history, work rationalisation, workshop

\section{AUTEUR}

\section{ÉRIC GEERKENS}

Historien, assistant à la Faculté d'économie, de gestion et des sciences sociales de l'université de Liège 